\title{
Efectos del manejo tradicional SOBRe la palma BRAHEA aculeata en una Selva SECA del sur de Sonora, MÉXico
}

\author{
Leonel Lopez-Toledo ${ }^{1,2,3}$, Carlos Espinosa-Hidalgo ${ }^{1}$, Christa Horn² y Bryan A. Endress ${ }^{2}$ \\ 'Instituto de Investigaciones sobre los Recursos Naturales, Universidad Michoacana de San Nicolás de Hidalgo, \\ Morelia, Michoacán, México \\ ${ }^{2}$ Division of Applied Plant Ecology, Institute for Conservation Research, San Diego Zoo Global, Escondido, \\ California, Estados Unidos de América \\ ${ }_{3}^{3}$ Autor para la correspondencia: leonellopeztoledo@gmail.com
}

\begin{abstract}
Resumen: En este estudio se evaluaron los efectos del manejo tradicional de Brahea aculeata (Arecaceae), sobre algunos atributos funcionales (hojas totales, producción y tamaño de hojas) y demográficos (mortalidad, crecimiento y reproducción). Las hojas de la especie son utilizadas para techos de casas y artesanías; y debido al pastoreo libre de ganado vacuno en el bosque, la especie puede sufrir herbivoría. Para evaluar los efectos del pastoreo y la cosecha de hojas se estableció un experimento en la Reserva "Sierra de Álamos", Sonora, México, en el que se simularon las diferentes prácticas del manejo tradicional. Se establecieron seis tratamientos que combinan el pastoreo (con/sin) e intensidades de cosecha (sin cosecha/baja/intensiva). En general, en palmas pequeñas ( $\leq 200 \mathrm{~cm}$ de largo de tallo), se encontraron efectos interactivos del pastoreo y la cosecha de hojas, mientras que en palmas grandes (> $200 \mathrm{~cm}$ ) únicamente para la cosecha. En palmas pequeñas se encontraron efectos negativos en el número y tamaño de hojas; mientras que la producción de hojas, la mortalidad y el crecimiento, el efecto fue positivo. Para palmas grandes, el efecto fue positivo en todos los casos; excepto en la mortalidad, en los que no se encontraron efectos. Los efectos positivos se podrían explicar como una respuesta sobrecompensatoria en la que la pérdida de área foliar se puede suplir mediante la alteración de procesos relacionados con la fotosíntesis y/o la asignación de recursos. Este estudio contribuye con información útil para el establecimiento de un programa de manejo, basado en el aprovechamiento tradicional de la especie en el área.
\end{abstract}

Palabras clave: atributos funcionales y demográficos, productos forestales no maderables, Reserva "Sierra de Álamos-Río Cuchujaqui"

\begin{abstract}
In this study, we evaluate the effects of traditional management of Brahea aculeata (Arecaceae) on certain functional (total, production and size of leaves) and demographic (mortality, growth and reproduction) attributes in a tropical dry forest in southern Sonora, Mexico. The leaves of this species are used for roofing houses and handcrafts. Also, in the area free-range cattle feed on palm leaves, especially in the dry season. To evaluate the effects of grazing and leaf harvest, an experiment was established in Reserva "Sierra de Álamos", Sonora, in which we simulated the different traditional management practices. We established six treatments that combined grazing (with/without) and harvest intensities (no harvest/low/intensive). In small palms ( $\leq 200 \mathrm{~cm}$ stem length) interactive effects were generally found between grazing and leaf harvest, while in large palms ( $>200 \mathrm{~cm}$ stem length) effects were only found for harvesting. In the small palms, negative effects were found in the number and size of leaves; while in leaf production, mortality and growth the effects were found to be positive. In the large palms, the effect of harvesting was positive in all cases, except for mortality, which did not present effects. The positive effects can be explained as an over compensatory response about of the loss of leaf area by the alteration of processes related to photosynthesis and/or the resource re-assignation. This study contributes valuable information for the establishment of an appropriate management program based on the traditional use of the species in the area.
\end{abstract}

Key words: functional and demographic attributes, non-timber forest products, Reserva Sierra de "Álamos-Río Cuchujaqui".

C on frecuencia se ha sugerido que el uso de los productos forestales no maderables (PFNM) es una buena opción ecológica y económica para la conservación y el aprovechamiento sustentable de recursos naturales (Pedersen y
Balslev, 1992; Putz et al., 2001). Para algunos de los PFNM, se ha demostrado que su uso es ecológicamente menos destructivo y puede permitir la permanencia de las poblaciones a largo plazo (Vasquez y Gentry, 1989; Caballero et al., 
2004; Coronel y Pulido, 2010). Además, su aprovechamiento puede contribuir a la subsistencia y bienestar de las personas que viven en o alrededor de los bosques (Endress et al., 2004; Pavón et al., 2006). En este sentido, es necesario estudiar los efectos del aprovechamiento sobre las especies que permita generar información ecológica robusta y que contribuya en el establecimiento de criterios de manejo más adecuados (Martínez-Ramos y Álvarez-Buylla, 1995; Olmsted y Álvarez-Buylla, 1995).

En la mayoría de las regiones tropicales del mundo, la familia Arecaceae representa una fuente de PFNM muy importante. Esta familia contiene muchas especies útiles que satisfacen muchas de las necesidades de las familias rurales (Pavón et al., 2006; Martínez-Ballesté et al., 2008). Además, debido a su comercialización llegan a representar, en ocasiones, un ingreso importante a la economía doméstica (Pedersen y Balslev, 1992; Velásquez. 1998; Pavón et al., 2006, Calvo-Irabien et al., 2009). Uno de los productos más importantes de las palmas son sus hojas, ya que se utilizan de una gran variedad de formas. Por ejemplo, las hojas de varias especies tales como Brahea, Chameadorea, Geonoma, Sabal y Thrinax, por mencionar algunas, sirven para la construcción de techos de casas, fabricación de artesanías, en la industria floral y hortícola (Caballero et al., 2004; Endress et al., 2004; Pavón et al., 2006; Martínez-Ballesté et al., 2008; Calvo-Irabien et al., 2009; Lopez-Toledo et al., 2011). Aún cuando algunos estudios documentan la sobreexplotación de las hojas de palma (O’Brieny Kinnaird, 1996) o la tala de individuos completos para facilitar su cosecha, los métodos de extracción en vegetación natural muy pocas veces involucran la destrucción de la planta (Peters et al., 1989; Vasquez y Gentry, 1989; Pedersen y Balslev, 1992; Pavón et al., 2006).

En términos generales, las palmas explotadas en vegetación natural han demostrado ser tolerantes a la defoliación y capaces de recuperarse, o no presentar efectos después de pocos eventos de cosecha de hojas y en bajas intensidades (Oyama y Mendoza, 1990; Zuidema et al., 2000; Coronel y Pulido, 2010). Algunos estudios indican que la cosecha de hojas a largo plazo puede estar relacionada con cambios fisonómicos en las poblaciones, tales como aumentos en la densidad, menor altura de los individuos y mayor producción de hojas (Pavón et al., 2006). Sin embargo, el efecto de la cosecha sobre atributos foliares y/o reproductivos, varía entre especies de palmas, así como de la intensidad y frecuencia con que sean cosechadas (Martínez-Ballesté et al., 2008, Calvo-Irabién et al., 2009). Por ejemplo, en poblaciones de Brahea dulcis bajo cosechas intensivas semestrales, se encontró un mayor crecimiento y producción de hojas que aquellas sin cosecha o cosecha baja (Coronel y Pulido, 2010). En contraste, poblaciones de Thrinax radiata sometidas a cosechas intensivas, sí presentaron efectos negativos sobre la producción y el tamaño de hojas (Calvo-Irabién et $a l ., 2009)$. Por otra parte, el libre pastoreo de ganado vacuno en la selva es una práctica muy común, que se caracteriza por la presencia de ganado en la selva durante todo el año, de manera rotacional o en algunos periodos de tiempo (Herrera, 1995). Durante estos periodos, el ganado puede alimentarse de pastos, especies arbóreas y en ocasiones palmas y agaves (Endress et al., 2004; Martín et al., 2011). Esto puede afectar y disminuir la regeneración natural de algunas especies, dado el ramoneo y pisoteo provocado por el ganado (Herrera, 1995). El efecto del ramoneo en las plantas puede ser más fuerte que la defoliación, debido a que el ganado puede aumentar la tasa de mortalidad, aunque esto depende del tamaño de las plantas (Endress et al., 2004). En general, las plántulas son las que tienen mayor probabilidad de morir al ser arrancadas del suelo al momento del ramoneo (Herrera, 1995; Vandenberghe et al., 2007; Martin et al., 2011; Lopez-Toledo et al., 2011).

Brahea aculeata es una especie endémica del Noroeste de México y se encuentra listada en la NOM-ECOL-059 (SEMARNAT, 2010). Estudios descriptivos de diferentes poblaciones en la Sierra de Álamos indican que la cosecha de hojas y el pastoreo puede tener daños sobre las demografía poblacional y puede estar contribuyendo a la disminución de la especie en el área (Lopez-Toledo et al., 2011). Sin embargo, hasta la fecha no se conoce con certeza los efectos directos de la cosecha de hojas y el pastoreo, sobre atributos funcionales y patrones demográficos de la especie. Este tipo de estudios son necesarios en la generación de recomendaciones de uso y conservación de la especie. Especialmente, esto resulta necesario, dado que representa una especie endémica de gran importancia ecológica y económica.

Considerando estos antecedentes, en el presente estudio se presenta la respuesta experimental de individuos de $\mathrm{Bra}$ hea aculeata al pastoreo y a la cosecha de hojas. Este experimento se llevó a cabo para explorar el efecto del manejo tradicional de la especie sobre algunos atributos funcionales y demográficos. Se espera que individuos sometidos a pérdida de área foliar provocada por el pastoreo y/o la cosecha de hojas tendrán un efecto negativo sobre los atributos funcionales y demográficos. Estos daños serán diferenciales dependiendo del tamaño de los individuos. Considerando esta hipótesis, las preguntas de este estudio fueron (1) ¿cuáles son los efectos de la cosechas de hojas sobre algunos atributos funcionales y demográficos de Brahea aculeata?, y (2) ¿existe un efecto diferencial del pastoreo y la cosecha de hojas dependiendo del tamaño de los individuos?

\section{Materiales y métodos}

Sitio de estudio. El estudio se llevó a cabo en el Área de Protección de Flora y Fauna "Sierra de Álamos-Rio $\mathrm{Cu}$ chujaqui" (APFF-SARC), que es un área natural protegida con 92,889 ha en el Estado de Sonora (Lopez-Toledo et al., 2011). El APFF-SARC forma parte de una red de áreas naturales protegidas reconocida por la UNESCO (PRO- 
MAB-UNESCO). Las elevaciones dentro de la reserva van desde los 300 hasta 1,600 m s.n.m., lo que da lugar a un gradiente de vegetación que va desde bosque tropical caducifolio a bosque de pino-encino y bosques de pino. Las precipitaciones anuales en la zona presentan una media de $650 \mathrm{~mm}$ (rango 190-1,120 mm), para el periodo 1970-2000. La estación seca es de ocho meses (noviembre a junio) y es muy pronunciada, recibiendo solo el $25-35 \%$ del total de precipitación anual. La temperatura media anual es de 21 a $22{ }^{\circ} \mathrm{C}$, con 10 y $41{ }^{\circ} \mathrm{C}$ como temperaturas mínimas y máximas, respectivamente. Este estudio se realizó en la cuenca alta del Rio Cuchujaqui, específicamente en una propiedad privada conocida como los Llanos $\left(27^{\circ} 03^{\prime} 51.27^{\prime \prime} \mathrm{N}, 108^{\circ}\right.$ $45^{\prime} 40.78$ " O).

Especie de estudio. Brahea aculeata (Brandegee) H.E.Moore (basiónimo de Erythea aculeata Brandegee) es una palma solitaria con reproducción hermafrodita. Tienen hojas palmeadas flabeladas con láminas y grandes inflorescencias (100-150 cm de longitud). Esta especie es endémica del Norte de México, específicamente de los estados de Chihuahua, Durango, Sinaloa y Sonora. Tiene una distribución irregular y pueden encontrarse en una amplia gama de hábitats, desde laderas soleadas montañosas hasta áreas más sombrías a lo largo de los arroyos, y en el fondo de cañones en el bosque tropical seco, bosques de encino y de pino-encino, entre los 300-1,500 m s.n.m. (Lopez-Toledo et al., 2011). En el bosque seco tropical del APFF-SARC, B. aculeata crece hasta $10 \mathrm{~m}$ de altura y $25 \mathrm{~cm}$ de diámetro (Quero, 2000; Felger $e t$ al., 2001). Se encuentra en la Lista Roja de la IUCN como "Vulnerable" (A1c) y como "Especie bajo Protección Especial" en la Lista Roja Mexicana (Quero, 1998; Felger et al., 2001; SEMARNAT, 2010).

Manejo tradicional. Las hojas de Brahea aculeata representan un recurso forestal no maderable muy importante en el noroeste de México (Joyal, 1996). Específicamente, sus hojas son utilizadas para la construcción de techos de casas y fabricación de artesanías. Pobladores del área indican que la cosecha de hojas de esta especie se ha llevado a cabo desde al menos hace 50 años (Lopez-Toledo et al., 2011). La cosecha de hojas, tanto para techado como para la fabricación de artesanías, se lleva a cabo principalmente durante los meses más fríos (diciembre-marzo), ya que es cuando, a decir de la gente local, las hojas son más suaves y permite su tejido. Desde hace algunos años existe una demanda muy importante de las hojas de $B$. aculeata para la construcción de palapas en la costa de Sonora. La cosecha de hojas se lleva a cabo tanto por pobladores locales (del interior de la reserva), como por pobladores externos (que vienen de ciudades más grandes, como Álamos, Navojoa y Ciudad Obregón). La cosecha entre estos dos grupos difiere principalmente en la cantidad de hojas cortadas de cada palma, el tamaño de las hojas y de las plantas cosechadas. La cosecha practicada por los pobladores locales es principalmente para autoconsumo, aunque ocasionalmente también se comercializa. Por el contrario, los pobladores externos cosechan las hojas principalmente para su comercialización. Los primeros cortan todas las hojas, excepto las últimas dos mas nuevas y aquellas que presenten alguna señal de herbivoría y/o algún daño. Por el contrario, los segundos cosechan todas las hojas disponibles independientemente de su calidad. Por otra parte, el tamaño de las plantas que se cosechan difiere entre estos dos grupos. En general, los primeros cosechan plantas $>10 \mathrm{~cm}$ de altura que presentan hojas con láminas $>60 \mathrm{~cm}$, mientras que los segundos pueden cosechar plantas desde $5 \mathrm{~cm}$ de altura y hojas desde $45 \mathrm{~cm}$ de largo. Respecto a lo cogollos (hojas meristemáticas), el primer grupo siempre deja al menos uno sin cosechar, mientras que los externos cosechan todos los cogollos disponibles.

Por otra parte, en el área de la Reserva "Sierra de Álamos", al ganado vacuno se le deja pastar libremente al interior de la selva, especialmente durante la época de secas. Esto, al parecer, provoca daños a las plantas debido al pisoteo y el ramoneo. Para el caso de Brahea aculeata, parece tener más efectos durante la época de secas debido a que, por su carácter perennifolio, es más buscada por el ganado vacuno para alimentarse de ella (Vásquez-León y Liverman, 2004; Lopez-Toledo et al., 2011). Contrario a otras especies de palmas, el manejo de B. aculeata no incluye el uso de fuego.

Diseño del experimento. El diseño de este estudio consiste en reproducir de manera sistemática y en lo máximo posible el manejo tradicional (pastoreo y cosecha de hojas) de Brahea aculeata en la Reserva "Sierra de Álamos-Río Cuchujaqui". Para esto, se establecieron seis cuadrantes de $625 \mathrm{~m}^{2}(25 \times$ $25 \mathrm{~m}$ ) en sitios con una alta abundancia de $B$. aculeata, separados por al menos $200 \mathrm{~m}$. El pastoreo se evaluó mediante sitios con pastoreo (P) y sin pastoreo (SP), mientras que la cosecha de hojas se evaluó con tres intensidades de cosecha: sin cosecha (control), cosecha baja (que representa la practicada por pobladores locales) y cosecha alta (que representa la practicada por pobladores externos). De la interacción de estos dos factores y sus niveles resultaron seis diferentes tratamientos. Cada tratamiento fue asignado al azar a uno de los seis cuadrantes. Las poblaciones han sido tradicionalmente sometidas a cosecha de hojas y pastoreo por ganado vacuno en un largo periodo (alrededor de 50 años); aunque previo al inicio del estudio, las poblaciones utilizadas tenían un año de no ser cosechadas. En general, en toda la APFF-SARC y la región de Álamos, no se conocen áreas sin manejo de la especie (Lopez-Toledo et al., 2011).

Los cuadrantes se marcaron con estacas y dentro de dicho cuadrante se registraron y etiquetaron todos los individuos de Brahea aculeata, incluyendo plántulas $\geq 1 \mathrm{~cm}$ de altura hasta adultos de $8 \mathrm{~m}$. Para los tratamientos de ausencia de ganado se construyó una cerca de alambre de púas para 
evitar su entrada, mientras que para el tratamiento con presencia de ganado se permitió el pastoreo. Específicamente, el ganado pastorea en el área donde se ubicaron los cuadrantes durante la época de mayor estiaje (abril a junio), que es cuando se presenta una escasez de forraje. La densidad de ganado en el sitio de estudio varía entre 1.0-1.5 cabezas ha ${ }^{-1}$. Debido a que $B$. aculeata es la única o de las pocas especies perennifolias, el ganado se alimenta de las hojas de la especie, aunque también los dueños del ganado suministran complementos alimenticios. Los tratamientos de cosecha de hojas se llevaron a cabo en diciembre de 2010, y se aplicaron de manera intensiva (CI), baja (CB) y sin cosecha (SC). Para cada individuo se registró la altura, diámetro a la base, número de hojas vivas, largo del peciolo y lámina de la hoja más nueva, número de inflorescencias e infrutescencias, número de frutos y reclutamiento de nuevos individuos (plántulas recién emergidas).

Análisis estadístico. El análisis se centró en la evaluación de los efectos del pastoreo y la cosecha de hojas sobre algunos atributos funcionales y demográficos de Brahea aculeata. Como un indicador de los atributos funcionales se utilizaron los siguientes atributos foliares: (1) hojas vivas, (2) producción de hojas nuevas y (3) largo de la lámina. En los atributos demográficos se incluyeron (1) mortalidad y (2) crecimiento (cuantificado como la tasa anual de extensión del tallo: $\mathrm{cm}$ ind. ${ }^{-1}$ año ${ }^{-1}$ ). No se realizaron análisis de atributos reproductivos y/o reclutamiento, ya que durante el año de estudio estos atributos tuvieron registros muy bajos.

Para analizar variables discretas de los atributos foliares, como son: hojas vivas, hojas muertas, producción de hojas y hojas meristemáticas, se utilizaron modelos lineares generalizados (GLM) utilizando un error de distribución Poisson. Para variables continuas (largo de lámina y crecimiento) se utilizaron análisis de varianza considerando una distribución normal, previa exploración de los supuestos de normalidad y homogeneidad de varianzas. La mortalidad se analizó como una variable binaria, para lo cual se utilizaron modelos lineares generalizados (GLM) utilizando un error de distribución binomial (Crawley, 2007).

Para identificar los posibles efectos del pastoreo y la cosecha de hojas de manera más evidente, se llevaron a cabo análisis por separado para plantas pequeñas y grandes. Las primeras se definieron como aquellas que potencialmente presentan los efectos de la cosecha de hojas y el pastoreo ( $\leq 200 \mathrm{~cm}$ de largo de tallo), mientras que las segundas fueron aquellas plantas que sufren solamente los efectos de la cosecha de hojas y que no son ramoneadas por el ganado ( $>200 \mathrm{~cm}$ de largo de tallo), aunque podrían presentar daños por otros efectos del pastoreo (pisoteo, compactación del suelo, etc).

Se llevaron a cabo análisis de covarianza utilizando tres variables explicativas: pastoreo (P: pastoreo y sin pastoreo), cosecha de hojas (C: sin cosecha, cosecha baja, cosecha in- tensiva) y el largo del tallo (LT). En el análisis para cada variable de respuesta, primero se produjeron modelos saturados que consideraron todos los factores y las interacciones posibles. Posteriormente, estos modelos se simplificaron al eliminarse las interacciones y/o términos no significativos (Crawley, 2007). Cuando la variable continua fue significativa, se obtuvo un modelo de la forma $y=m x+b$, donde $m$ es la pendiente y $b$ la ordenada al origen. Se probaron diferencias entre las pendientes entre los tratamientos que resultaron significativos. Las líneas de regresión presentadas en los resultados fueron obtenidas de estos modelos. Todos los análisis se llevaron a cabo en el programa estadístico $\mathrm{R}$ 2.11.1 (R Development Core Team, 2010).

\section{Resultados}

Los individuos bajo tratamiento de pastoreo y cosecha de hojas, presentaron diferencias respecto a las palmas control. Estas diferencias dependen de los atributos analizados; en algunos casos fueron positivos, lo que indica un mejor desempeño que plantas control, aunque también se detectaron efectos negativos sobre algunos de los atributos analizados. Además, estos efectos variaron dependiendo del tamaño de los individuos. Especialmente, se observó que plantas pequeñas fueron afectadas tanto por pastoreo como por la cosecha de hojas, mientras que plantas grandes fueron únicamente afectadas por la cosecha de hojas. A continuación se presentan los resultados, primero para atributos funcionales y después para los demográficos. En cada caso se describen por separado los efectos en plantas pequeñas $(\leq 200 \mathrm{~cm}$ de largo de tallo) y después para plantas grandes (>200 cm de largo de tallo).

Atributos funcionales. El número de hojas vivas en pie en plantas pequeñas presentó una alta variación (rango: 1-20 hojas) y se encontró una relación positiva con el largo del tallo. Esta relación fue diferente entre tratamientos de pastoreo y cosecha, lo que resultó en una interacción significativa entre estos dos factores. El análisis de covarianza indicó que el tratamiento control (SP-SC) presentó mayor pendiente $(m=6$ hojas $\mathrm{m}^{-1}$ ); es decir, individuos bajo pastoreo y cualquier tratamiento de cosecha, presentaron un menor número de hojas que el tratamiento control (Cuadro 1, Figura 1). Para plantas grandes solo se encontraron efectos por la cosecha de hojas. Contrario a plantas pequeñas, el tratamiento con mayor pendiente fue la cosecha intensiva $\left(m=2\right.$ hojas $\left.\mathrm{m}^{-1}\right)$, lo que indica una mayor cantidad de hojas vivas que en los otros tratamientos, especialmente para individuos $>400 \mathrm{~cm}$ de largo de tallo. El pastoreo por ganado no afectó la cantidad de hojas vivas en estos individuos (Cuadro 1, Figura 1).

La producción de hojas nuevas en palmas pequeñas varió entre 0 y 16 hojas y también se encontró una relación positiva entre el número de hojas producidas y el tamaño de la planta. Esta relación varió entre los diferentes trata- 


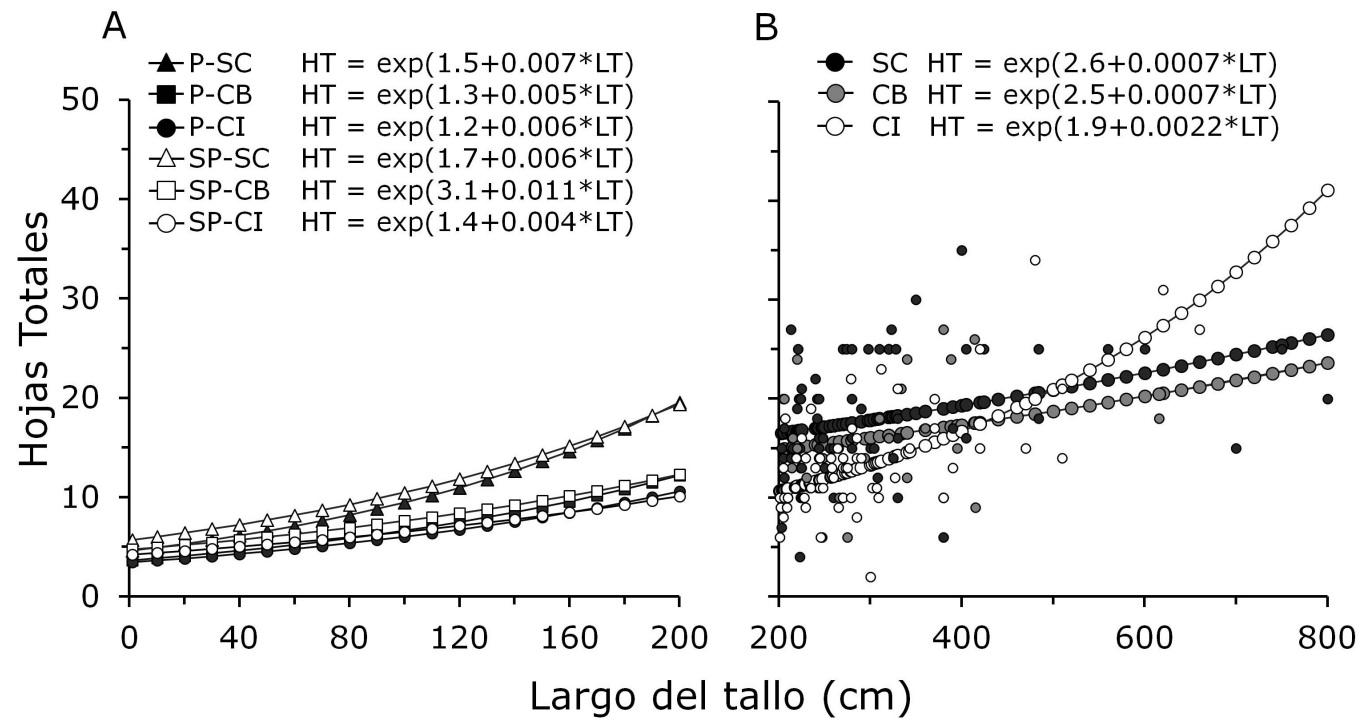

Figura 1. Número de hojas totales en individuos de Brahea aculeata sometidos a diferentes tratamientos de pastoreo (P: pastoreo y S: sin pastoreo) y cosecha de hojas (SC: sin cosecha, CB: cosecha baja y CI: cosecha intensiva) en el APFF-SARC, Sonora, México. Las gráficas muestran los resultados del análisis para A: plantas pequeñas $(<200 \mathrm{~cm})$ y B: grandes $(>200 \mathrm{~cm})$. Se presentan las ecuaciones y las líneas de regresión obtenidas con base en los modelos lineares generalizados con distribución Poisson. Para A no se presentan los valores

observados debido a la gran cantidad de individuos y para mayor claridad en la gráfica. Notar las diferentes escalas en Y.

mientos de cosecha y pastoreo. El tratamiento de pastoreo y cosecha baja fue el que mayor tasa de producción de hojas presentó ( $m=6$ hojas $\mathrm{m}^{-1}$; Cuadro 1, Figura 2). Para palmas grandes también se registró una relación con el tamaño del individuo, aunque esta relación varió entre tratamientos de cosecha. En este caso, se presentó una mayor producción de hojas en palmas cosechadas, ya sea con baja ( $m=2$ hojas $\left.\mathrm{m}^{-1}\right)$ o intensa cosecha $\left(m=1\right.$ hoja $\left.\mathrm{m}^{-1}\right)$. Tampoco se detectó un efecto del pastoreo sobre la producción de hojas (Cuadro 1, Figura 2).

En general, el largo de la lámina de hojas nuevas en palmas pequeñas varió entre 83.2 y $110 \mathrm{~cm}$. El análisis estadístico indicó diferencias dependiendo del tamaño de los indivi- duos y entre los tratamientos de cosecha de hojas, aunque no entre palmas con y sin pastoreo. Palmas sin cosecha presentaron una mayor pendiente $\left(m=1.1 \mathrm{~cm} \mathrm{~cm}^{-1}\right)$, lo que indica láminas de mayor tamaño, mientras que palmas cosechadas tanto a baja como alta intensidad produjeron láminas más pequeñas. Especialmente las palmas más grandes (190-200 $\mathrm{cm}$ ) tuvieron, según el modelo, una reducción de 10 y $20 \%$ respecto a individuos control que presentaron hojas de 105 cm de largo (Cuadro 1, Figura 3). Para el caso de palmas grandes también se encontró una relación significativa entre el largo de la hoja y el largo del tallo. Esta relación varió entre los diferentes tratamientos de cosecha de hojas, obteniendo diferentes ordenadas al origen y pendientes. El trata-

Cuadro 1. Efectos de diferentes niveles de cosecha de hojas y pastoreo sobre atributos foliares de plantas pequeñas $(\leq 200 \mathrm{~cm})$ y grandes $(>200 \mathrm{~cm})$ de Brahea aculeata en la Reserva "Sierra de Álamos-Río Cuchujaqui", Sonora, México. Se presentan los resultados de los análisis de covarianza bajo un modelo linear generalizado (GLM) para hojas vivas (HV) y producción de hojas (PH), y un modelo lineal (LM) para largo de la hoja nueva (LH). Se presentan los estadísticos para los GLM y LM (valor de $\chi^{2}, F$ y grados de libertad, respectivamente). Nivel de significancia: $* P<0.05$; ${ }^{* *} P<0.01 y^{* * *} P<0.001$, ns indica que el término no fue significativo.

\begin{tabular}{|c|c|c|c|c|c|c|c|c|c|c|c|c|}
\hline \multirow{3}{*}{$\begin{array}{l}\text { Variables } \\
\text { Factores }\end{array}$} & \multicolumn{6}{|c|}{ Plantas pequeñas $(<200 \mathrm{~cm})$} & \multicolumn{6}{|c|}{ Plantas grandes $(>200 \mathrm{~cm})$} \\
\hline & \multicolumn{2}{|c|}{ HV } & \multicolumn{2}{|c|}{$\mathrm{PH}$} & \multicolumn{2}{|c|}{$\mathrm{LH}$} & \multicolumn{2}{|c|}{ HV } & \multicolumn{2}{|l|}{$\mathrm{PH}$} & \multicolumn{2}{|c|}{$\mathrm{LH}$} \\
\hline & $\chi^{2}$ & g.l. & $\chi^{2}$ & g.l & $\mathrm{F}$ & g.l & $\chi^{2}$ & g.l & $\chi^{2}$ & g.l & $\mathrm{F}$ & g.l \\
\hline Largo de tallo (LT) & $729.5^{* * *}$ & 1 & $60.5^{* * *}$ & 1 & $29.1^{* * *}$ & 1,1090 & $59.4^{* * *}$ & 1 & $30.01^{* * *}$ & 1 & $20.9^{* * *}$ & 1,157 \\
\hline Pastoreo (P) & $76.5^{* * *}$ & 1 & $5.1^{* *}$ & 1 & $0.7^{\mathrm{ns}}$ & 1,1090 & $1.6^{\mathrm{ns}}$ & 1 & $5.3^{\text {ns }}$ & 1 & $1.5^{\mathrm{ns}}$ & 1,157 \\
\hline Cosecha de hojas $(\mathrm{CH})$ & $80.5^{* * *}$ & 2 & $9.1^{* *}$ & 2 & $9.3^{* * *}$ & 2,1090 & $39.1^{* * *}$ & 2 & $17.5^{* * *}$ & 2 & $0.81^{\mathrm{ns}}$ & 2,157 \\
\hline LT:P & $4.6^{* *}$ & 1 & $13.9^{* * *}$ & 1 & $1.9^{\text {ns }}$ & 1,1090 & $1.2^{\mathrm{ns}}$ & 1 & $4.1^{\mathrm{ns}}$ & 1 & $0.9^{\text {ns }}$ & 1,157 \\
\hline LT:CH & $7.3^{* *}$ & 2 & 1.4 & 2 & $7.9^{* * *}$ & 2,1090 & $17.9^{* * *}$ & 2 & $5.8^{*}$ & 2 & $11.8^{* * *}$ & 2,157 \\
\hline $\mathrm{P}: \mathrm{CH}$ & $20.0^{* * *}$ & 2 & $14.4^{* * *}$ & 2 & $3.2^{\mathrm{ns}}$ & 2,1090 & $7.5^{\mathrm{ns}}$ & 2 & $3.9^{\text {ns }}$ & 2 & $1.4^{\mathrm{ns}}$ & 2,157 \\
\hline LT:P:CH & $1.9^{\text {ns }}$ & 2 & $11.7^{* *}$ & 2 & $2.6^{\mathrm{ns}}$ & 2,1090 & $4.6^{\mathrm{ns}}$ & 2 & $0.7^{\mathrm{ns}}$ & 2 & $1.9^{\mathrm{ns}}$ & 2,157 \\
\hline
\end{tabular}


A

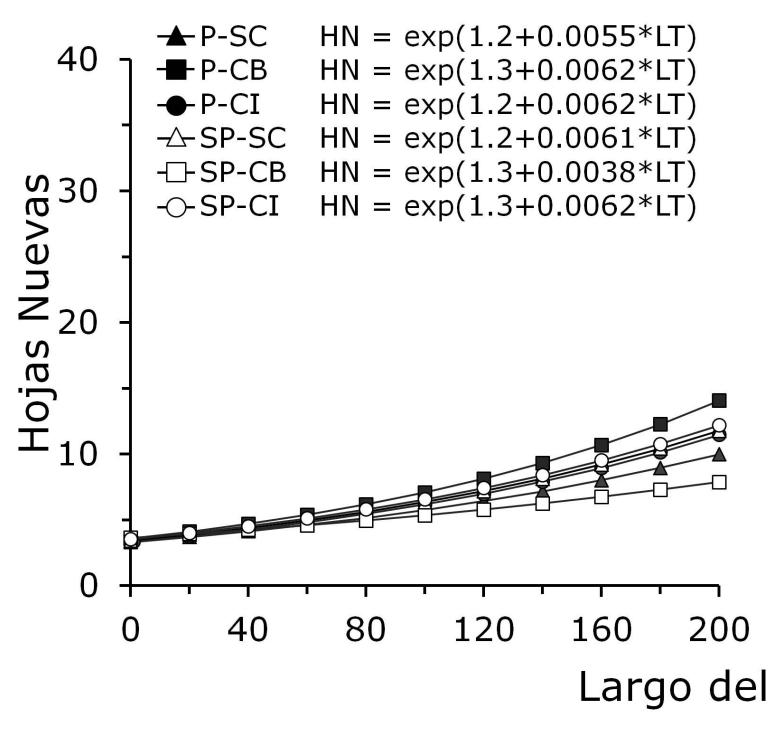

B

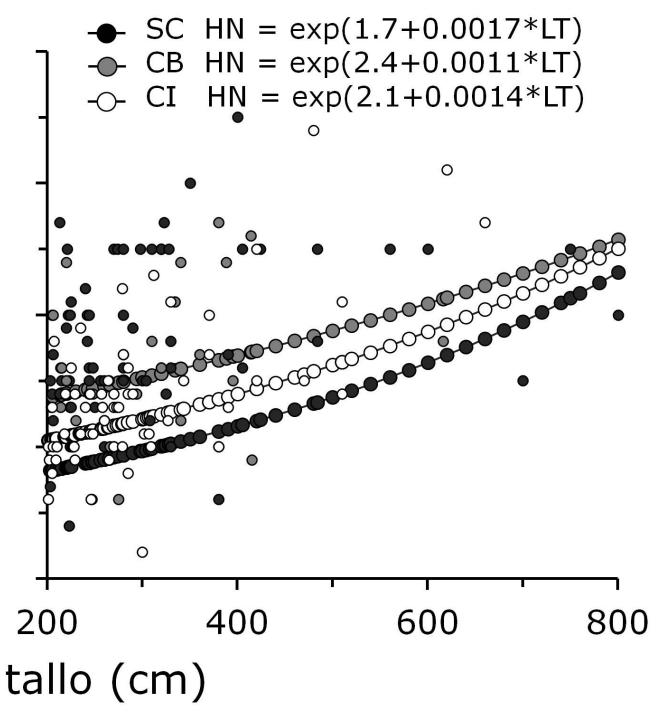

Figura 2. Producción de hojas nuevas en individuos de Brahea aculeata sometidos a diferentes tratamientos de pastoreo y cosecha de hojas en el APFF-SARC, Sonora, México (ver figura 1 para equivalencias de los tratamientos). Las gráficas muestran los resultados del análisis para A: plantas pequeñas $(\leq 200 \mathrm{~cm})$ y B: grandes $(>200 \mathrm{~cm})$. Se presentan las ecuaciones y las líneas de regresión obtenidas con base en los modelos lineares generalizados con distribución Poisson. Para A no se presentan los valores observados debido a la gran cantidad de individuos y para mayor claridad en la gráfica.

miento de cosecha baja fue el que mayor tamaño de lámina presentó en función de su tamaño (Cuadro 1, Figura 3).

Atributos demográficos. Para palmas pequeñas, los resultados obtenidos muestran que la mortalidad varió de acuerdo al tamaño. En palmas sin pastoreo la mortalidad aumentó con el largo del tallo; mientras que en palmas que tuvieron pastoreo, sorpresivamente, la mortalidad disminuyó con el tamaño (Cuadro 2, Figura 4). Para el caso de palmas grandes, se registró una mortalidad muy baja con alrededor de 1-2 plantas muertas por tratamiento, por lo que no se llevó a cabo ningún análisis estadístico.

En el crecimiento en tallo de palmas pequeñas se encontró que este no depende del tamaño de los individuos. Los

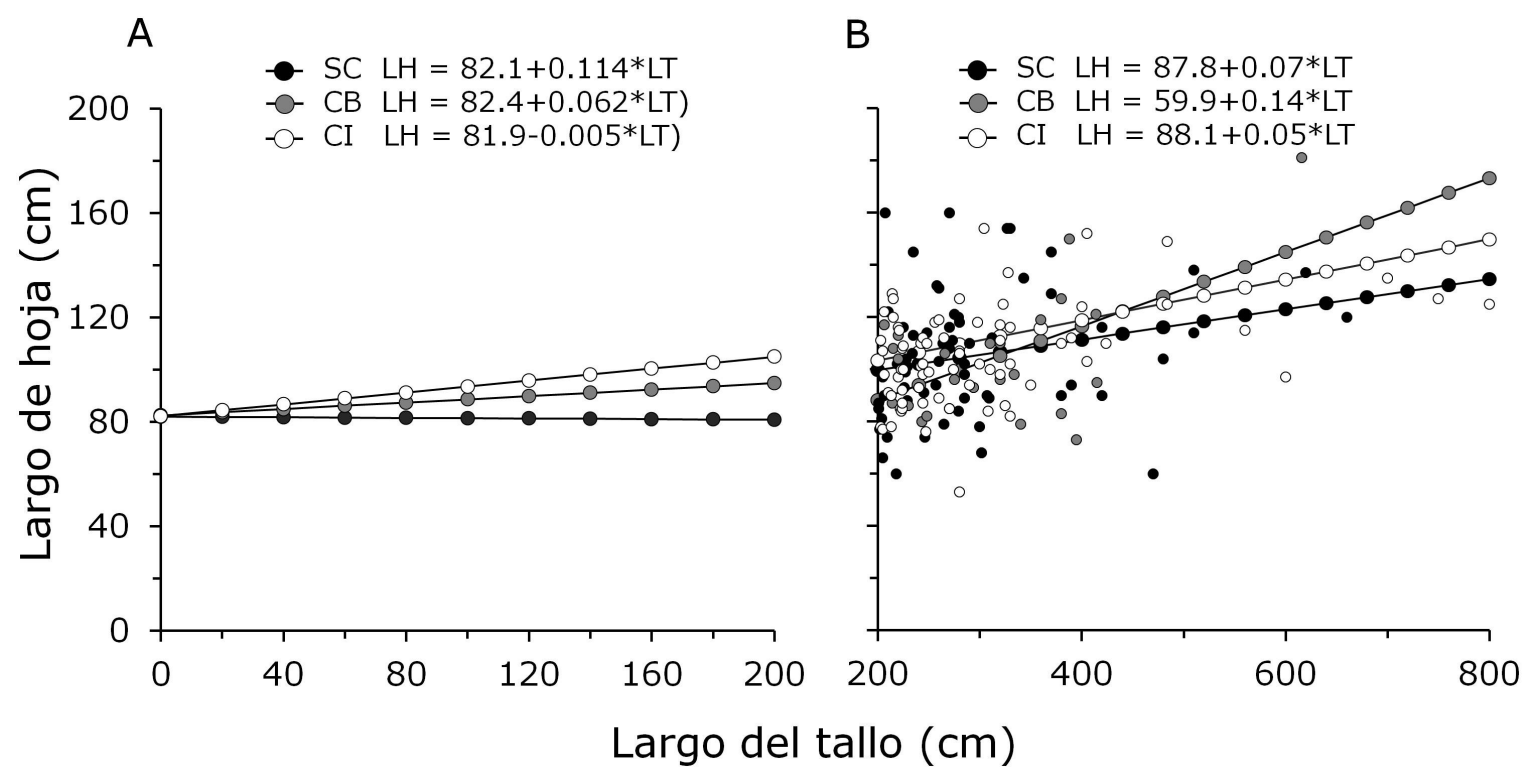

Figura 3. Largo de la hoja nueva en individuos de Brahea aculeata sometidos a diferentes tratamientos de pastoreo y cosecha de hojas en el APFF-SARC, Sonora, México (ver figura 1 para equivalencias de los tratamientos). Las gráficas muestran los resultados del análisis para A: plantas pequeñas $(\leq 200 \mathrm{~cm})$ y B: grandes $(>200 \mathrm{~cm})$. Para A se presentan las líneas de regresión obtenidas a partir de los modelos lineares generalizados con distribución Poisson. 


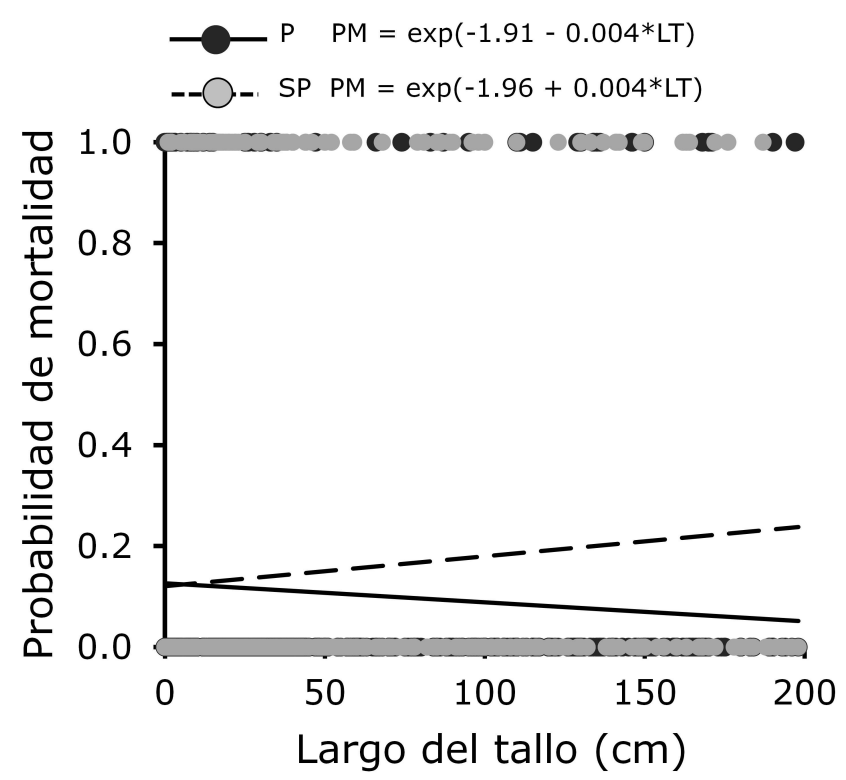

Figura 4. Probabilidad de mortalidad de individuos de Brahea aculeata sometidos a diferentes tratamientos de pastoreo y cosecha de hojas en el APFF-SARC, Sonora, México (ver figura 1 para equivalencias de los tratamientos). La gráfica presentada muestran los resultados del análisis para plantas pequeñas $(\leq 200$ $\mathrm{cm})$. Las líneas representan la regresión logística obtenida con base en un modelo lineal generalizado con distribución binomial (ver cuadro 2).

únicos factores significativos fueron el pastoreo y la cosecha de hojas. Sorpresivamente, se registró un mayor crecimiento en plantas con pastoreo y cosecha intensiva (promedio \pm error estándar $=6.8 \pm 1.1 \mathrm{~cm}$ año $0^{-1}$ ), aunque palmas con cosecha baja y sin cosecha presentaron un crecimiento entre 50 y $55 \%$ menor, respectivamente. En palmas sin pastoreo se observó un crecimiento de entre $2.3( \pm 0.3)$ y $4.4( \pm 0.6)$ $\mathrm{cm}$ año-1 para aquellas con cosecha intensiva y sin cosecha, respectivamente (Cuadro 2, Figura 5). Para palmas grandes, el crecimiento fue independiente del tamaño del individuo y el análisis no detectó un efecto significativo del pastoreo. Por el contrario, la cosecha de hojas incrementó el crecimiento significativamente, especialmente para palmas bajo cosecha intensiva (promedio \pm error estándar $=10.7 \pm 1.2$ $\mathrm{cm}$ año ${ }^{-1}$ ). El menor crecimiento se encontró en el tratamiento de cosecha baja (Cuadro 2, Figura 5).

\section{Discusión}

Varios estudios han descrito que el pastoreo de manera extensiva y permanente, incluso a densidades de ganado moderadas $\left(0.7\right.$ cabezas ha $\left.{ }^{-1}\right)$ pueden tener fuertes efectos sobre la demografía de varias especies de plantas (Dufour-Dror, 2007). Específicamente se ha detectado una reducción importante de la sobrevivencia y el desarrollo de plántulas de árboles, arbustos y palmas, que afecta no solamente los patrones demográficos y la regeneración de las especies, sino también al ecosistema en general (Distel et al., 1996; Endress et al., 2004; Dufour-Dror, 2007). En el caso de Brahea aculeata, los resultados permiten sugerir que el pastoreo no tiene fuertes efectos negativos sobre la especie, ya que estos únicamente se detectaron sobre dos de los cinco atributos analizados (hojas totales y producción de hojas nuevas) en palmas pequeñas. En los otros atributos analizados, los efectos fueron positivos.

Se ha descrito que la presencia de ganado disminuye la emergencia y/o establecimiento de plántulas al ser ramoneadas y/o pisoteadas (Pulido y Díaz, 2005). En caso de que las plántulas o brinzales sobrevivan al efecto del ramoneo o pisoteo provocado por el ganado, estas interrumpen severamente su desarrollo, ya que la planta permanece con área foliar insuficiente para un desempeño eficiente (Distel et al., 1996; Pulido y Díaz, 2005). También se ha mencionado que el pastoreo podría tener efectos positivos sobre la regeneración de algunas especies debido a que el ramoneo

Cuadro 2. Efectos de diferentes niveles de cosecha de hojas y pastoreo sobre parámetros demográficos de plantas pequeñas ( $\leq 200 \mathrm{~cm})$ y grandes (> $200 \mathrm{~cm}$ ) de Brahea aculeata en la Reserva "Sierra de Álamos-Río Cuchujaqui", Sonora, México. Se presentan los resultados de los análisis de covarianza bajo un modelo linear generalizado (GLM) con distribución binomial para mortalidad y un modelo lineal (LM) para el crecimiento. Se presentan los estadísticos para los GLM y LM (valor de $\chi^{2}, \mathrm{~F}$ y grados de libertad, respectivamente). Nivel de significancia: ${ }^{*} P<0.05$; ${ }^{* *} P<$ $0.001 y^{* * *} P<0.001$, ns indica que el término no fue significativo. Para la mortalidad en palmas grandes no se realizó ningún modelo, debido al bajo número de individuos muertos.

\begin{tabular}{|c|c|c|c|c|c|c|c|c|}
\hline \multirow{3}{*}{$\begin{array}{l}\text { Variables } \\
\text { Factores }\end{array}$} & \multicolumn{4}{|c|}{ Plantas pequeñas $(<200 \mathrm{~cm})$} & \multicolumn{4}{|c|}{ Plantas grandes $(>200 \mathrm{~cm})$} \\
\hline & \multicolumn{2}{|c|}{ Mortalidad } & \multicolumn{2}{|c|}{ Crecimiento } & \multicolumn{2}{|c|}{ Mortalidad } & \multicolumn{2}{|c|}{ Crecimiento } \\
\hline & $\chi^{2}$ & g.l & $F$ & g.l & $\chi^{2}$ & g.l & $F$ & g.l \\
\hline Largo del tallo (LT) & 0.004 & 1 & $12.2 * * *$ & 1,542 & - & - & $0.02^{\mathrm{ns}}$ & 1,61 \\
\hline Pastoreo (P) & $6.2 * *$ & 1 & $0.32^{\mathrm{ns}}$ & 1,542 & - & - & $0.05^{\mathrm{ns}}$ & 1,61 \\
\hline Cosecha de hojas $(\mathrm{CH})$ & $1.1^{\mathrm{ns}}$ & 2 & $0.4^{\mathrm{ns}}$ & 2,542 & - & - & $3.59^{* *}$ & 2,61 \\
\hline LT:P & $7.0^{* * *}$ & 1 & $1.9^{\mathrm{ns}}$ & 2,542 & - & - & $1.4^{\mathrm{ns}}$ & 1,61 \\
\hline $\mathrm{LT}: \mathrm{CH}$ & $1.2^{\mathrm{ns}}$ & 2 & $1.4^{\mathrm{ns}}$ & 2,542 & - & - & $0.4^{\mathrm{ns}}$ & 2,61 \\
\hline $\mathrm{P}: \mathrm{CH}$ & $0.8^{\text {ns }}$ & 2 & $8.2^{* * *}$ & 2,542 & - & - & $0.01^{\mathrm{ns}}$ & 2,61 \\
\hline $\mathrm{LT}: \mathrm{P}: \mathrm{CH}$ & $1.4^{\mathrm{ns}}$ & 2 & $0.7^{\mathrm{ns}}$ & 2,542 & - & - & $0.2^{\mathrm{ns}}$ & 2,61 \\
\hline
\end{tabular}


A

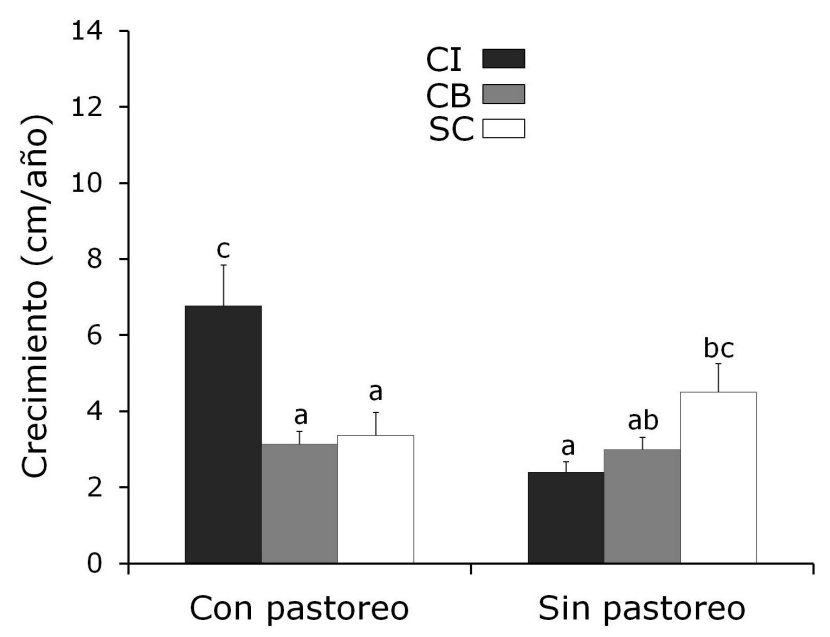

B

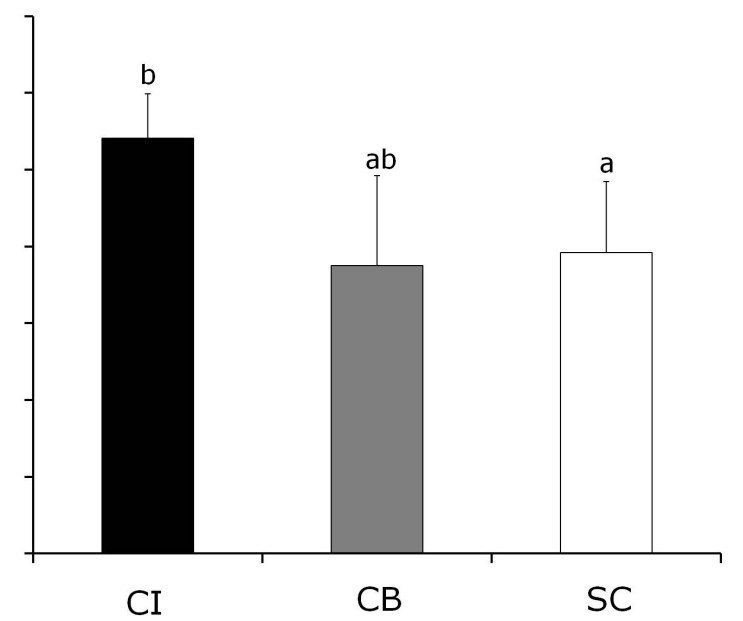

Figura 5. Crecimiento en individuos de Brahea aculeata sometidos a diferentes tratamientos de pastoreo y cosecha de hojas en el APFFSARC, Sonora, México (ver figura 1 para equivalencias de los tratamientos). Las gráficas muestran los resultados del análisis para A: plantas pequeñas $(\leq 200 \mathrm{~cm})$ y B: grandes $(>200 \mathrm{~cm})$.

del estrato herbáceo podría reducir la competencia (Distel et al., 1996).

El efecto del ramoneo en palmas ha sido muy poco evaluado y hasta le fecha, el único estudio en palmas es con la especie Chamaedorea radicalis en bosques mesófilos de Tamaulipas (Endress et al., 2004). En ese estudio se encontró que la exposición de plantas al ramoneo incrementa significativamente la mortalidad de todos los estadios de vida y la tasa de crecimiento, incluso con un solo evento de ramoneo. Las bajas tasas de crecimiento de las poblaciones de esta especie fueron el resultado de las tasas de mortalidad de plántulas, juveniles y adultos pequeños (Endress et al., 2004). En el presente estudio, con Brahea aculeata se encontró una menor mortalidad y mayor crecimiento, al menos en individuos bajo cosecha intensiva, en los tratamientos bajo pastoreo. Este aparente efecto positivo es un resultado que contradice la hipótesis original y se debe de tomar con precaución. Los sitios de muestreo, y en general toda el área alrededor, utilizados para el experimento han sido manejados con pastoreo y cosecha de hojas durante mucho tiempo (al menos 50 años; Lopez-Toledo et al., 2011). Esto, al parecer, ha provocado una reducción en la densidad de plantas del sotobosque. El experimento de exclusión de ganado permitió la proliferación masiva de plantas del estrato herbáceo de hasta 1.5-2.0 m de altura. Probablemente, esto impide el acceso de luz hasta el sotobosque y, por lo tanto, podría impedir la captación de esta por parte de palmas menores a este tamaño. Además, la proliferación de estas hierbas puede aumentar la competencia entre raíces en el suelo, lo que potencialmente le impediría a las palmas captación de nutrientes y agua (Gill y Marks, 1991; Distel et al., 1996). Por el contrario, en los cuadrantes con pastoreo, a pesar de que también surge una gran cantidad plantas en el estrato herbáceo, el pisoteo por el ganado provoca su mortalidad. Como resultado de esto se podría esperar mayor acceso de luz hasta el sotobosque y menor competencia entre raíces, lo que permitiría a las palmas una mejor captación de nutrientes y agua; por lo tanto, una mejor supervivencia y crecimiento, como se ha sugerido en estudios previos (Gill y Marks, 1991; Distel et al., 1996). Por otra parte, también se ha demostrado que el suelo de áreas con pastoreo presenta una mayor compactación, lo que podría impedir el desarrollo del sistema radical de las plántulas y por lo tanto, un menor crecimiento o mayor mortalidad (Distel et al., 1996); sin embargo en este estudio no se encontró dicho efecto.

Parece ser que el mejor diseño de un experimento para evaluar los efectos del pastoreo y cosecha de hojas podría ser utilizando poblaciones de palmas que nunca hayan estado sometidas a estos dos factores; sin embargo, según nuestro conocimiento en la región, estas poblaciones no existen en el área debido al manejo tradicional de la especie durante mucho tiempo (Lopez-Toledo et al., 2011). Para el caso de plantas grandes, se presentó una mortalidad muy baja con alrededor de 1-2 plantas muertas por tratamiento, lo que podría indicar que el pastoreo no tiene efectos negativos sobre la mortalidad de estas palmas. Como se supone, las plantas más grandes escapan al efecto del ramoneo y por lo tanto no se esperaría ningún daño sobre los individuos, por lo que puede pensarse que la cosecha de hojas es el factor más importante. Sin embargo, se necesitan estudios a largo plazo para evaluar de una manera más contundente el efecto del ganado sobre esta especie, ya que un año de estudio puede no estar reflejando los efectos acumulados.

Al parecer, el impacto de la cosecha de hojas depende del gremio ecológico de la especie, así como de la intensidad y la frecuencia con que se lleve a cabo la cosecha. Para 
especies de palmas de sotobosque (Chamaedorea) de selvas húmedas, se ha descrito que una cosecha de baja intensidad con un solo evento o con periodos largos entre cosechas tendrá un bajo o nulo impacto e incluso, hasta respuestas positivas (Oyama y Mendoza 1990; Martínez-Ramos et al., 2009). Por el contrario, aquellos individuos sometidos a intensidades altas de cosecha y con periodos de descanso cortos producirá mayores efectos negativos (Martínez-Ramos et al., 2009), lo cual se observa en la disminución de parámetros reproductivos, seguidos por el crecimiento $\mathrm{y}$, si la cosecha persiste, un aumento de la mortalidad (Lopez-Toledo et al., 2012). Para el caso de especies similares a la de este estudio, como por ejemplo Brahea dulcis, de ambientes secos con alta intensidad lumínica y que alcanzan el dosel, los efectos hasta ahora descritos indican un bajo o nulo impacto sobre la demografía de la especie (Coronel y Pulido 2010). Sin embargo, para Thrinax radiata un sólo evento de cosecha, a intensidad muy alta (100\%), si afectó la producción y el tamaño de hojas (Calvo-Irabién, 2009).

En el presente estudio no se encontró una fuerte reducción (o incluso la respuesta fue positiva) en los atributos analizados. Es altamente probable que esto se debió a que únicamente se realizó un solo evento de cosecha. Al considerar los resultado de otros estudios, se esperaría que si la cosecha persiste se presentarían efectos negativos más intensos (Anten et al., 2003; Martínez-Ramos et al., 2009; Lopez-Toledo et al., 2012).

Con respecto a los atributos foliares analizados, las palmas cosechadas presentaron una mejor respuesta comparadas con las palmas control. Especialmente las palmas grandes tuvieron valores más altos, tanto en los atributos foliares, así como en el crecimiento. Por el contrario, las palmas pequeñas bajo cosecha tuvieron una respuesta menor respecto a las palmas control. Estas respuestas positivas y que representan un mejor desempeño que el grupo control, pudieron deberse a un efecto de sobrecompensación (Crawley, 1983). Este proceso se ha descrito ya en otras especies bajo experimentos similares de cosecha de hojas (Anten et al., 2003). Cuando plantas defoliadas tienen un desempeño similar al que se esperaría por predicción o comparados con plantas no dañadas (plantas control) se dice que hay una compensación. Si el desempeño de plantas dañadas es mayor, se dice que hay una sobrecompensación y por el contrario, cuando el desempeño es menor que las plantas control, se dice que hay un subcompensación (Bergelson et al., 1996; Strauss y Agrawal, 1999; Anten et al., 2003). En el caso de Brahea aculeata es posible que, como un efecto de sobrecompensación, las palmas cosechadas a mayor intensidad hayan tenido un mayor crecimiento.

Respecto a los atributos reproductivos, no se realizó ningún análisis, ya que durante el año de seguimiento se registró una muy baja reproducción. En una evaluación rápida en distintas poblaciones de Brahea aculeata con diferente manejo, al relacionar la cantidad de hojas cosechadas y la probabilidad de reproducción, se encontró una correlación negativa. Es decir, la reproducción es menor con el aumento en el área foliar perdida (Lopez-Toledo et al., 2011). Sin embargo, en el presente estudio no se encontró este efecto, debido a la baja reproducción, incluso en el grupo control. Específicamente se reprodujeron entre 0 y 2 palmas por tratamiento $(<1 \%)$, con un total de ocho palmas reproductivas. En el experimento de defoliación mencionado anteriormente sobre Chamaedorea radicalis, se encontró que en general las poblaciones tienen una baja reproductividad, con alrededor de $9 \%$ de la población control en reproducción (Endress et al., 2004). Sin embargo, la pérdida de área foliar disminuye aún más esta reproducción, alcanzándose hasta proporciones de $1 \%$ según la intensidad de defoliación. En otro estudio, C. elegans con defoliaciones semestrales permanentes durante tres años, se encontró que dependiendo de la intensidad de defoliación la reproducción disminuye. Aún más, este efecto se va intensificando con el número de cosechas, tanto en la probabilidad de reproducción, como en la producción de infrutescencias y número de frutos (Martínez-Ramos et al., 2009), y todavía después de tres años de descanso, las poblaciones defoliadas con mayor intensidad no se recuperan (Lopez-Toledo et al., 2012). Para especies similares a $B$. aculeata aún no existen estudios de los efectos de la cosecha de hojas sobre el componente reproductivo. Lo observado en $B$. aculeata pudo deberse al manejo que se le ha dado a la especie durante mucho tiempo (al menos 50 años), ya que es muy extraño que incluso plantas del grupo control hayan tenido una muy baja reproducción.

\section{Conclusiones}

En este estudio se determinaron los efectos del manejo tradicional sobre diferentes atributos foliares y demográficos en la palma Brahea aculeata. El pastoreo y la cosecha de hojas tienen efectos negativos sobre algunos atributos de la especie; sin embargo, ambos provocan una respuesta positiva en algunos atributos foliares y del crecimiento, lo que se podría explicarse como una respuesta sobrecompensatoria (Anten et al., 2003).

En palmas con cosecha de hojas se encontró una disminución en el tamaño de hojas. Se encontró también que los efectos son diferenciales dependiendo del tamaño de los individuos. Así, individuos pequeños fueron los más afectados por la cosecha de hojas y el pastoreo. Los resultados de este estudio deben de ser tomados con precaución, pues representan un año de seguimiento demográfico y los efectos a largo plazo pueden ser diferentes, por lo que es necesario llevar a cabo este tipo de estudios a largo plazo. Este estudio contribuye con información ecológica, basada en el manejo tradicional, que puede ser utilizada para el establecimiento de un programa de aprovechamiento de Brahea aculeata que permita un uso racional de esta especie de palma endémica del noroeste de México. 


\section{Agradecimientos}

Este estudio fue apoyado por Lakeside Foundation y San Diego Zoo Global. Nature and Culture International y su personal apoyaron en el trabajo de campo. Agradecemos al Sr. Jesús Álvarez las facilidades para llevar a cabo el experimento en el Rancho Los Llanos. También, agradecemos a la APFF-Sierra de Álamos-Río Cuchujaqui y la DGVSSEMARNAT por las facilidades y los permisos concedidos (No. SGPA/DGVS/01991/10 y SGPA/DGVS/10652/10). Agradecemos al editor y los revisores anónimos por sus sugerencias y comentarios que mejoraron sustancialmente el artículo.

\section{Literatura citada}

Anten N.P.R., Martínez-Ramos M. y Ackerly D.D. 2003.Defoliation and growth in an understory palm: quantifying the contributions of compensatory responses. Ecology 84:2905-2918.

Bergelson J., Juenger T. y Crawley M.J. 1996. Regrowth following herbivory in Ipomopsis aggregata: compensation but no overcompensation. American Naturalist 148:744-755.

Caballero J., Pulido M.T. y Martínez-Ballesté A. 2004. El uso de la palma de guano (Sabal yapa) en la industria turística de Quintana Roo, Mexico. En: Alexiades M.N. y Shanley P. Eds. Productos Forestales, Medios de Subsistencia y Conservacion. Estudios de Caso sobre Sistemas de Manejo de Productos Forestales No Maderables. Vol. 3., pp. 365-385. Centro para la Investigación Forestal Internacional, Bogor Barat.

Calvo-Irabién L.M., Zapata M.T. y Iriarte-Vivar S. 2009. Effects of leaf harvest on Thrinax radiata palm: implication for management and conservation. Journal of Tropical Forest Science 21:34-44.

Coronel O.M. y Pulido S.M.T. 2010. Uso artesanal, fenología y cosecha óptima de la palma Brahea dulcis (Kunth) Mart. en el estado de Hidalgo, México. En: Moreno F.A., Pulido S.M.T., Mariaca M.R., Valadez A.R., Mejía C.P. y Gutiérrez S.T.V. Eds. Sistemas Biocognitivos Tradicionales. Paradigmas en la Conservación Biológica y el Fortalecimiento Cultural, pp. 24-29, Asociación Etnobiológica Mexicana A.C., Global Diversity Foundation, Universidad Autónoma del Estado de Hidalgo, El Colegio de la Frontera Sur, Sociedad Latinoamericana de Etnobiología, México.

Crawley M.J. 1983. Herbivory: The Dynamics of Animal-Plant Interactions. University of California Press, Berkeley.

Crawley M.J. 2007. The R Book. John Wiley \& Sons Ltd., Chichester.

Distel R.A., Peláez D.V., Bóo R.M., Mayor M.D. y Elía O.R. 1996. Growth of Prosopis caldenia seedlings in the field as related to grazing history of the site and in a greenhouse as related to different levels of competition from Stipa tenuis. Journal of Arid Environments 32:251-257.

Dufour-Dror J.M. 2007. Influence of cattle grazing on the density of oak seedlings and saplings in a Tabor oak forest in Israel. Acta Oecologica 31:223-238.

Endress B.A., Gorchov D.L. y Noble R.B. 2004. Non-timber forest product extraction: effects of harvest and browsing on an understory palm. Ecological Applications 14:1139-1153.
Felger R.S., Johnson M.B. y Wilson M.F. 2001. The Trees of Sonora, Mexico. Oxford University Press, Nueva York.

Gill D.S. y Marks P.L. 1991. Tree and shrub seedling colonization of old fields in central New York. Ecological Monographs 61:183-205.

Herrera J. 1995. Acorn predation and seedling production in a lowdensity population of cork oak (Quercus suber L.). Forest Ecology and Management 76:197-201.

Joyal E. 1996. The use of Sabal uresana (Arecaceae) and other palms in Sonora, Mexico. Economic Botany 50:429-445.

Lopez-Toledo L., Anten N.P.R., Endress B.A., Ackerly D.D. y Martínez-Ramos M. 2012. Resilience to chronic defoliation in a dioecious understorey tropical rain forest palm. Journal of Ecology 100:1245-1256.

Lopez-Toledo L., Horn C. y Endress B.A. 2011. Distribution and population patterns of the threatened palm Brahea aculeata in a tropical dry forest in Sonora, Mexico. Forest Ecology and Management 261:1901-1910.

Martin M.P., Peters C.M., Palmer M.I. y Illsley C. 2011. Effect of habitat and grazing on the regeneration of wild Agave cupreata in Guerrero, Mexico. Forest Ecology and Management 262:1443-1451.

Martínez-Ballesté A., Martorrel C. y Caballero J. 2008. The effect of Maya traditional harvesting on the leaf production and demographic parameters of Sabal palm in the Yucatán Peninsula, Mexico. Forest Ecology and Management 256:1320-1324.

Martínez-Ramos M. y Alvarez-Buylla E. 1995. Ecología de poblaciones de plantas en una selva húmeda de México. Boletín de la Sociedad Botánica de México 56:121-153.

Martínez-Ramos M., Anten N.P.R. y Ackerly D.D. 2009. Defoliation and ENSO effects on vital rates of an understorey tropical rain forest palm. Journal of Ecology 97:1050-1061.

O'Brien T.G. y Kinnaird M.F. 1996. Effect of harvest on leaf development of Asian palm Livistona rotundifolia. Conservation Biology 10:53-58.

Olmsted I. y Álvarez-Buylla E. 1995. Sustainable harvesting of tropical trees: demography and matrix models of two palm species in México. Ecological Applications 5:484-500.

Oyama K. y Mendoza A. 1990. Effects of defoliation on growth, reproduction, and survival of a neotropical dioecious palm, Chamaedorea tepejilote. Biotropica 22:119-123.

Pavón N.P., Escobar R.I. y Ortiz-Pulido R. 2006. Extracción de hojas de la palma Brahea dulcis en una comunidad otomí en Hidalgo, México: efecto sobre algunos parámetros poblacionales. Interciencia 31:57-61.

Pedersen H.B. y Balslev H. 1992. The economic botany of Ecuadorean Palms. En: PlotkinM.J.. y Famolare L.M. Eds. Sustainable Harvest and Marketing of Rain Forest Products, pp. 173-192, Island Press, Washington, D.C.

Peters C.M., Gentry A.H. y Mendelsohn R.O. 1989. Valuation of an Amazonian Rainforest. Nature 339:655-656.

Pulido F.J. y Díaz M. 2005. Regeneration of a mediterranean oak: a whole-cycle approach. Ecoscience 12:92-102.

Putz F.E., Blate G.M., Redford K.H., Fimbel R. y Robinson J. 2001. Tropical forest management and conservation of biodiversity: an overview. Conservation Biology 15:7-20.

Quero R.H.J. 1998. Brahea aculeata. IUCN Red List of Threatened Species. Version 2010.2. <http://www.iucnredlist.org/details/38454/0> (consultado el 16 de Julio 2015).

Quero R.H.J. 2000. El complejo BraheaErythea (Palmae: 
Coryphoideade). Informe final SNIB-CONABIO proyecto No. L216. Instituto de Biología, Universidad Nacional Autónoma de México, México.

R Development Core Team. 2010. R: A language and environment for statistical computing. R Foundation for Statistical Computing. <www.r-project.org> (consultado 15 de Febrero de 2013).

SEMARNAT [Secretaría del Medio Ambiente y Recursos Naturales]. 2010. Norma Oficial Mexicana NOM-059-SEMARNAT-2010, Protección ambiental - Especies nativas de México de flora y fauna silvestres - Categorías de riesgo y especificaciones para su inclusión, exclusión o cambio - Lista de especies en riesgo. Diario Oficial de la Federación. 2da Sección, 30 de diciembre de 2010.

Strauss S.Y. y Agrawal A.A. 1999. The ecology and evolution of plant tolerance to herbivory. Trends in Ecology and Evolution 14:179-185.
Vasquez-Leon M. y Liverman D. 2004. The political ecology of land-use change: affluent ranchers and destitute farmers in the Mexican municipio of Álamos. Human Organization 63:21-33.

Vasquez R. y Gentry A.H. 1989. Use and misuse of forest-harvested fruits in the Iquitos area. Conservation Biology 3:350-361.

Velásquez R.J. 1998. Productivity and sustainability of a vegetable ivory palm (Phytelephas aequatorialis, Arecaceae) under three management regimes in northwestern Ecuador. Economic Botany 52:168-182.

Vandenberghe C., Freléchoux F., Moravie M.A., Gadallah F. y Buttler A. 2007. Short-term effects of cattle browsing on tree sapling growth in mountain wooded pastures. Plant Ecology 188:253-264.

Zuidema P.A. 2000. Demography of Exploited Tree Species in the Bolivian Amazon. Programa Manejo de Bosques de la Amazonia Boliviana, Universiteit Utrecht, Utrecht.

Recibido: 5 de marzo de 2014

Aceptado: 24 de septiembre de 2014 